(1)

CrossMark

\title{
Treatment with inhaled $\alpha 1$-antitrypsin: a square peg in a round hole?
}

\author{
Miriam Barrecheguren and Marc Miravitlles (D)
}

Affiliation: Pneumology Dept, Hospital Universitari Vall d'Hebron/Vall d'Hebron Research Institute (VHIR), Barcelona, Spain.

Correspondence: Marc Miravitlles, Pneumology Dept, Hospital Universitari Vall d'Hebron, P. Valld'Hebron 119-129. 08035 Barcelona, Spain. E-mail: marcmasepar.es

@ERSpublications

Inhaled $\alpha 1$-antitrypsin may reduce severity of exacerbations in COPD http://bit.ly/2OtJx $8 \mathrm{~b}$

Cite this article as: Barrecheguren M, Miravitlles M. Treatment with inhaled $\alpha 1$-antitrypsin: a square peg in a round hole?. Eur Respir J 2019; 54: 1901894 [https://doi.org/10.1183/13993003.01894-2019].

a1-Antitrypsin deficiency (AATD) is a genetic disorder that predisposes to the development of early pulmonary emphysema, especially in smokers. Episodes of exacerbations are frequent in patients with emphysema due to AATD and are associated with a deficient antiprotease screen in the airways compared with that of non-deficient COPD patients [1]. As a consequence, exacerbations have great impact on the evolution of the lung disease in AATD, measured in terms of decline in gas transfer [2], in health status $[2,3]$, and in lung function over time $[4,5]$.

To date, the only specific treatment for AATD-related emphysema is the intravenous infusion of purified $\alpha 1$-antitrypsin (AAT) derived from plasma donors, so-called augmentation therapy. Previous randomised clinical trials (RCTs) have consistently shown the efficacy of augmentation therapy in slowing the progression of pulmonary emphysema measured by computed tomography densitometry in individuals with severe AATD $[6,7]$. However, evidence of the effect of augmentation on exacerbations is very limited and comes exclusively from a couple of observational studies that described a reduction in the frequency of exacerbations in patients after initiation of therapy $[8,9]$. The lack of effect of augmentation therapy on exacerbations observed in RCTs can be due to different reasons: 1) the studies were not powered for exacerbations; 2) the patient populations were not enriched for exacerbators; and 3) augmentation per se may not have an effect on the prevention of exacerbations, but may help to preserve lung integrity in case of an exacerbation.

Due to this lack of evidence from RCTs of the effect of augmentation on secondary outcomes such as exacerbations, dyspnoea or quality of life, this therapy is still not reimbursed in some countries [10]. This unfortunate situation is a consequence of the erroneous approach of evaluating the efficacy of an aetiological or disease-modification treatment (augmentation therapy) with the same outcome measurements as a symptomatic treatment (bronchodilators). Why should an intravenous antiprotease reduce exacerbation frequency or improve dyspnoea or the St George's Respiratory Questionnaire score? The objective of augmentation therapy is to prevent or stop the evolution of emphysema, and this has been already demonstrated in the existing RCTs $[8,9,11]$.

However, treatment with AAT does not lack drawbacks. Therapy with AAT implies weekly intravenous infusions, it is time consuming and expensive, and is usually for life. Hence, there is interest in finding new treatments that may help reduce the progression and burden of the disease and that may be more convenient for patients. In this context, previous studies have shown that aerosol administration of AAT to 
patients with AATD was safe and feasible and resulted in a return to normal of anti-neutrophil elastase defences of the lower respiratory tract [12]. Inhaled AAT can also decrease the levels of elastase activity, neutrophils and pro-inflammatory cytokines in patients with cystic fibrosis [13] and can even decrease bacterial load in the respiratory tract in animal models [14].

Based on these observations, STOLK et al. [15] designed a double-blind RCT that aimed to demonstrate the effect of nebulised AAT on the reduction of exacerbations in patients with AATD. Both groups received 50 weeks of inhaled AAT or placebo twice a day. The results of the study showed no effect of AAT on time to first exacerbation compared to placebo. However, patients treated with inhaled AAT had a significant reduction in Anthonisen type I exacerbations (more symptomatic) and, interestingly, there was also a trend towards an improvement in forced expiratory volume in $1 \mathrm{~s}\left(\mathrm{FEV}_{1}\right)$ in the treatment arm [15].

The first observation is that, despite being well designed and conducted, the trial encountered some issues that could have hindered the efficacy of therapy. The nebuliser device and medication dispensation were improved along the study based on feedback from patients, which significantly reduced the rate of adverse events in the AAT group by the end of the study [15]. Given the high percentage of adverse events and withdrawals, and considering that some adverse events may have mimicked exacerbations, it seems reasonable to think that having an optimised treatment from the beginning would have had a positive effect on the results of efficacy of inhaled AAT.

The selection of exacerbations as a primary outcome can pose a challenge of its own. Since there are different phenotypes of exacerbations, and emphysematous patients may experience pauci-inflammatory episodes [16, 17], the possible benefits of the antiprotease effect on the prevention of exacerbations in AATD are not clear. In fact, among those patients presenting at least one exacerbation during the trial (85\%), almost half suffered a type III exacerbation (only one symptom), an increase in dyspnoea being the only symptom in around $80 \%$. It is very unlikely to see an impact of an antiprotease treatment on exacerbations characterised only by an increase in dyspnoea captured in a diary. However, STоLк et al. [15] also observed a decrease of type I exacerbations with inhaled AAT and a reduction of type II exacerbations, particularly those characterised by increased sputum volume and purulence. These results suggest that the maximal effect of this therapy could be expected in patients with an infectious exacerbator phenotype similar to cystic fibrosis, or in those with associated bronchiectasis [18].

Another important consideration is patient selection. Patients were included if they had had at least two exacerbations, either moderate or severe, during the previous 18 months, with one having taken place in the last 12 months. This definition does not guarantee that patients suffered from frequent exacerbations, and in fact $25 \%$ of patients did not fulfil the standard definition of a frequent exacerbator, which may have lessened the margin of improvement with treatment [15]. Nonetheless, the difficulties in recruiting enough individuals with a particular phenotype in a rare disease such as AATD have to be recognised (i.e. frequent exacerbators), and, therefore, the authors must be acknowledged for trying their best to enrich their population with exacerbators as much as possible.

Unexpectedly, patients treated with inhaled AAT showed a rapid increase in $\mathrm{FEV}_{1}$ of less than $50 \mathrm{~mL}$ after 4 weeks of treatment and maintained a mean difference of around $50 \mathrm{~mL}$ in $\mathrm{FEV}_{1}$ throughout the study compared to placebo. Further analysis of $\mathrm{FEV}_{1}$ decline without the data from the first 4 weeks, during which there was a "hockey stick effect" in the AAT group, suggested that the slope of decline of $\mathrm{FEV}_{1}$ might be favourable to the AAT arm. It should be noted that $\mathrm{FEV}_{1}$ was not even a secondary outcome and was evaluated only as a safety parameter and, therefore, these results should be interpreted with caution [15].

This pattern of improvement in lung function with inhaled AAT is similar to that observed with drugs such as inhaled corticosteroids (ICS) [19] or roflumilast [20], and suggests a local anti-inflammatory effect. Based on these findings, the authors defend the design of a new RCT with inhaled AAT using FEV 1 as the primary outcome. This is a very controversial proposal, because the alpha community has been struggling for decades to convince healthcare providers and regulators that, unlike non-AATD related $\mathrm{COPD}, \mathrm{FEV}_{1}$ is not an appropriate end-point for therapeutic trials in AATD, since the number of patients required to power a study for $\mathrm{FEV}_{1}$ decline is unattainable in this rare disease [21]. Furthermore, lung densitometry has shown to be a much better parameter to assess both the evolution of emphysema and the efficacy of treatment in AATD $[11,22,23]$. Insisting again on efficacy trials in AATD with FEV $_{1}$ as primary end-point is a dangerous strategy that will very likely produce negative results and increase the confusion among patients, physicians and payers. In the best-case scenario, an improvement in $\mathrm{FEV}_{1}$ within the range of less than $100 \mathrm{~mL}$ can also be obtained with other drugs such as ICS, which are less expensive and more convenient than inhaled AAT [24]. A possible lack of (or small) effect of AAT on $\mathrm{FEV}_{1}$ should not mislead us from recognising the crucial effect of augmentation therapy in reducing the rate of destruction of lung parenchyma in emphysema associated with AATD [25-27]. 
The authors must be congratulated for designing and conducting an RCT that represents a completely new approach in the treatment of patients with emphysema due to AATD. This study was highly anticipated, and although the results were not as positive as we had all hoped, it has provided very valuable information. The trial has demonstrated that the inhaled route of administration of AAT is safe and feasible and has improved the inhalation device and technique of administration for subsequent studies. Although the results obtained do not support the use of inhaled AAT for the majority of patients with emphysema associated with AATD, they also suggest that a subgroup of patients, those with frequent infective exacerbations, and that present with increased sputum volume and purulence, may benefit from inhaled AAT in the form of a change to a milder type of episodes and probably even with increased protection against the long-term effects of recurrent exacerbations in the lungs. Future studies with this type of treatment approach should focus on this specific phenotype of AATD individuals and investigate whether this effect justifies the long-term use of twice daily inhaled AAT on top of optimised COPD therapy.

Conflict of interest: M. Barrecheguren has received speaker fees from Grifols, Menarini, CSL Behring, GSK and consulting fees from GSK, Novartis and GebroPharma. M. Miravitlles reports speaker's fees from AstraZeneca, Boehringer Ingelheim, Chiesi, Cipla, Menarini, Rovi, Bial, Zambon, CSL Behring, Grifols and Novartis, consulting fees from AstraZeneca, Boehringer Ingelheim, Chiesi, GlaxoSmithKline, Bial, Gebro Pharma, CSL Behring, Laboratorios Esteve, Ferrer, Mereo Biopharma, Verona Pharma, TEVA, pH Pharma, Novartis and Grifols, and research grants from GlaxoSmithKline and Grifols, outside the submitted work.

\section{References}

1 Hill AT, Campbell EJ, Bayley DL, et al. Evidence for excessive bronchial inflammation during an acute exacerbation of chronic obstructive pulmonary disease in patients with alpha(1)-antitrypsin deficiency (PiZ). Am J Respir Crit Care Med 1999; 160: 1968-1975.

2 Needham M, Stockley RA. Exacerbations in alpha1-antitrypsin deficiency. Eur Respir J 2005; 25: 992-1000.

3 Bernhard N, Lepper PM, Vogelmeier C, et al. Deterioration of quality of life is associated with the exacerbation frequency in individuals with alpha-1-antitrypsin deficiency - analysis from the German Registry. Int J Chron Obstruct Pulm Dis 2017; 12: 1427-1437.

4 Hiller AM, Piitulainen E, Jehpsson L, et al. Decline in $\mathrm{FEV}_{1}$ and hospitalized exacerbations in individuals with severe alpha-1 antitrypsin deficiency. Int J Chron Obstruct Pulm Dis 2019; 14: 1075-1083.

5 Esquinas C, Serreri S, Barrecheguren M, et al. Long-term evolution of lung function in individuals with Alpha-1 Antitrypsin Deficiency from the Spanish Registry. Int J Chron Obstruct Pulm Dis 2018; 13: 1001-1007.

6 Chapman KR, Burdon JG, Piitulainen E, et al. Intravenous augmentation treatment and lung density in severe alpha-1 antitrypsin deficiency (RAPID): a randomized, double-blind, placebo-controlled trial. Lancet 2015; 386: 360-368.

7 Edgar RG, Patel M, Bayliss S, et al. Treatment of lung disease in alpha-1 antitrypsin deficiency: a systematic review. Int J Chron Obstruct Pulm Dis 2017; 12: 1295-1308.

8 Barros-Tizon JC, Torres ML, Blanco I, et al. Reduction of severe exacerbations and hospitalization-derived costs in alpha-1-antitrypsin-deficient patients treated with alpha-1-antitrypsin augmentation therapy. Ther Adv Respir Dis 2012; 6: 67-78.

9 Lieberman J. Augmentation therapy reduces frequency of lung infections in antitrypsin deficiency: a new hypothesis with supporting data. Chest 2000; 118: 1480-1485.

10 Horváth I, Canotilho M, Chlumský J, et al. Diagnosis and management of $\alpha_{1}$-antitrypsin deficiency in Europe: an expert survey. ERJ Open Res 2019; 5: 00171-2018.

11 Miravitlles M, Dirksen A, Ferrarotti I, et al. European Respiratory Society statement: diagnosis and treatment of pulmonary disease in alpha-1 antitrypsin deficiency. Eur Respir J 2017; 50: 1700610.

12 Hubbard RC, Brantly ML, Sellers SE, et al. Anti-neutrophil-elastase defenses of the lower respiratory tract in alpha 1-antitrypsin deficiency directly augmented with an aerosol of alpha 1-antitrypsin. Ann Intern Med 1989; 111: 206-212.

13 Griese M, Latzin P, Kappler M, et al. Alpha1-Antitrypsin inhalation reduces airway inflammation in cystic fibrosis patients. Eur Respir J 2007; 29: 240-250.

14 Cantin AM, Woods DE. Aerosolized prolastin suppresses bacterial proliferation in a model of chronic Pseudomonas aeruginosa lung infection. Am J Respir Crit Care Med 1999; 160: 1130-1135.

15 Stolk J, Tov N, Chapman KR, et al. Efficacy and safety of inhaled $\alpha 1$-antitrypsin in patients with severe $\alpha 1$-antitrypsin deficiency and frequent exacerbations of COPD. Eur Respir J 2019; 54: 1900673.

16 Bafadhel M, McKenna S, Terry S, et al. Acute exacerbations of chronic obstructive pulmonary disease: identification of biologic clusters and their biomarkers. Am J Respir Crit Care Med 2011; 184: 662-671.

17 Vogelmeier CF, Chapman KR, Miravitlles M, et al. Exacerbation heterogeneity in COPD: subgroup analyses from the FLAME study. Int J Chron Obstruct Pulm Dis 2018; 13: 1125-1134.

18 Parr DG, Guest PG, Reynolds JH, et al. Prevalence and impact of bronchiectasis in alpha1-antitrypsin deficiency. Am J Respir Crit Care Med 2007; 176: 1215-1221.

19 Burge PS, Calverley PM, Jones PW, et al. Randomised, double blind, placebo controlled study of fluticasone propionate in patients with moderate to severe chronic obstructive pulmonary disease: the ISOLDE trial. BMJ 2000; 320: 1297-1303.

20 Yuan L, Dai X, Yang M, et al. Potential treatment benefits and safety of roflumilast in COPD: a systematic review and meta-analysis. Int J Chron Obstruct Pulm Dis 2016; 11: 1477.

21 Schluchter MD, Stoller JK, Barker AF, et al. Feasibility of a clinical trial of augmentation therapy for alpha (1)-antitrypsin deficiency. The Alpha 1-Antitrypsin Deficiency Registry Study Group. Am J Respir Crit Care Med 2000; 161: 796-801. 
22 Stolk J, Putter H, Bakker EM, et al. Progression parameters for emphysema: a clinical investigation. Respir Med 2007; 101: 1924-1930.

23 Dowson LJ, Guest PJ, Stockley RA. Longitudinal changes in physiological, radiological, and health status measurements in alpha(1)-antitrypsin deficiency and factors associated with decline. Am J Respir Crit Care Med 2001; 164: 1805-1809.

24 Low EV, Hughes SM, Zaffarullah S, et al. ICS use may modify FEV1 decline in $\alpha 1$-antitrypsin deficiency patients with relatively high blood eosinophils. Respiration 2018; 95: 114-121.

25 Barrecheguren M, Miravitlles M. Augmentation therapy for emphysema due to alpha-1 antitrypsin deficiency: Pro. Arch Bronconeumol 2018; 54: 363-364.

26 Balbi B, Ferrarotti I, Miravitlles M. Efficacy of augmentation therapy for emphysema associated with $\alpha 1$-antitrypsin deficiency: enough is enough. Eur Respir J 2016; 47: 35-38.

27 Greulich T. Alpha-1-antitrypsin deficiency: disease management and learning from studies. COPD 2017; 14: Suppl. 1, S8-S11. 